\title{
HÖRMANDER CONDITIONS FOR VECTOR-VALUED KERNELS OF SINGULAR INTEGRALS AND THEIR COMMUTATORS
}

\author{
ANDREA L. GALLO, GONZALO H. IBAÑEZ FIRNKORN, \\ AND MARÍA SILVINA RIVEROS
}

\begin{abstract}
We study Coifman type estimates and weighted norm inequalities for singular integral operators and their commutators, given by the convolution with a vector-valued kernel. We define a weaker Hörmander type condition associated with Young functions for the vector-valued kernels. With this general framework we obtain as an example the result for the square operator and its commutator given in [M. Lorente, M. S. Riveros, and A. de la Torre, J. Math. Anal. Appl. 336 (2007), no. 1, 577-592].
\end{abstract}

\section{INTRODUCTION}

A classical problem in harmonic analysis is the following: given a linear operator $\mathcal{T}$, find the maximal operator $\mathcal{M}_{\mathcal{T}}$ such that $\mathcal{T}$ is controlled by $\mathcal{M}_{\mathcal{T}}$ in the following sense:

$$
\int_{\mathbb{R}^{n}}|\mathcal{T} f|^{p}(x) w(x) d x \leq C \int_{\mathbb{R}^{n}}\left|\mathcal{M}_{\mathcal{T}} f\right|^{p}(x) w(x) d x,
$$

for some $0<p<\infty$ and some $0 \leq w \in L_{\text {loc }}^{1}\left(\mathbb{R}^{n}\right)$.

The maximal operator $\mathcal{M}_{\mathcal{T}}$ is related to the operator $\mathcal{T}$ which is normally easier to deal with. In general, $\mathcal{M}_{\mathcal{T}}$ is strongly related to the kernel of $\mathcal{T}$.

The classical result of Coifman in [3] is: Let $T$ be a Calderón-Zygmund operator; then $T$ is controlled by $M$, the Hardy-Littlewood maximal operator. In other words, for all $0<p<\infty$ and $w \in A_{\infty}$,

$$
\int_{\mathbb{R}^{n}}|T f|^{p}(x) w(x) d x \leq C \int_{\mathbb{R}^{n}}(M f)^{p}(x) w(x) d x .
$$

Later in [16, Rubio de Francia, Ruiz, and Torrea studied operators with less regularity in the kernel. They proved that for certain operators, 1.1) holds with $\mathcal{M}_{\mathcal{T}}=M_{r} f=M\left(|f|^{r}\right)^{1 / r}$, for some $1 \leq r<\infty$. The value of the exponent $r$ is determined by the smoothness of the kernel, namely, the kernel satisfies an $L^{r^{\prime}}$-Hörmander condition (see the precise definition below). In [14], Martell, Pérez,

2010 Mathematics Subject Classification. 42B20, $42 \mathrm{~B} 25$.

Key words and phrases. Calderón-Zygmund operators; Commutators; BMO; Hörmander condition of Young type; Muckenhoupt weights; Vector-valued inequalities.

The authors are partially supported by CONICET and SECYT-UNC. 
and Trujillo-González proved that this control is sharp in the sense that one cannot write a pointwise smaller operator $M_{s}$ with $s<r$. This yields that for operators satisfying only the classical Hörmander condition, $H_{1}$, the inequality (1.1) does not hold for any $M_{r}, 1 \leq r<\infty$.

More recently, in [12], Lorente, Riveros, and de la Torre defined a $L^{\mathcal{A}}$-Hörmander condition where $\mathcal{A}$ is a Young function. If $T$ is an operator that satisfies this condition, then 1.1 holds for $M_{\overline{\mathcal{A}}}$, the maximal operator associated to the Young function $\overline{\mathcal{A}}$.

As a consequence of the Coifman inequality, a weighted modular end-point estimate can be proved. In [11, Lorente, Martell, Riveros, and de la Torre proved the following: if $\overline{\mathcal{A}}$ is submultiplicative and $\lambda>0$, then

$$
w\left\{x \in \mathbb{R}^{n}:|T f(x)|>\lambda\right\} \leq c \int_{\mathbb{R}^{n}} \overline{\mathcal{A}}\left(\frac{|f(x)|}{\lambda}\right) M w(x) d x .
$$

An example of this type of operator is the square operator $S$ (see the precise definition below); by the results in [12] the following inequality holds:

$$
\int_{\mathbb{R}^{n}}|S f|^{p}(x) w(x) d x \leq C \int_{\mathbb{R}^{n}}\left(M^{3} f\right)^{p}(x) w(x) d x,
$$

for all $0<p<\infty$ and $w \in A_{\infty}$.

In [13] it was proved that the last inequality is not sharp, in the sense that $M^{3}$ can be replaced by $M^{2}$.

In this paper we define a new Hörmander condition in the case of vector-valued kernels, weaker than the $L^{\mathcal{A}}$-Hörmander condition defined in [11]. We obtain inequality 1.1 improving results, for vector-valued operators, obtained in [1]. The applications of these results with the new condition are generalizations of those for the square operator obtained in [13. In these applications, the maximal operators are of the form $M_{L \log L^{\beta}}$, with some $\beta \geq 0$. For instance, we obtain for all $0<p<$ $\infty$ and $w \in A_{\infty}$,

$$
\int_{\mathbb{R}}\left|S_{X} f\right|^{p}(x) w(x) d x \leq C \int_{\mathbb{R}}\left(M^{2} f\right)^{p}(x) w(x) d x,
$$

where $X$ is an appropriate Banach space. If $X=\ell^{2}$, then $S_{X}=S$ the square operator, and in this case we obtain the same results as in [13.

In [2], Bernardis, Lorente, and Riveros defined $L^{\mathcal{A}, \alpha}$-Hörmander conditions for fractional integral operators. The authors obtained the inequality 1.1 with $M_{\overline{\mathcal{A}}, \alpha}$, the fractional maximal operator associated to $\overline{\mathcal{A}}$. In this paper, we also give a weaker condition for vector-valued kernels than the $L^{\mathcal{A}, \alpha}$-Hörmander condition and obtain a similar kind of results and applications.

The plan of this paper is as follows. The next section contains some definitions and well known results. Later, in section 3, we introduced our condition and the main results. The applications are presented in section 4 . The proofs of the general results are in sections 5. Finally in the last section we present the Hörmander condition and the results for vector-valued fractional operators. 


\section{Preliminaries}

In this section we present some notions needed to understand the main results and the applications. First we define the space in which we are going to work.

Let us consider the Banach spaces $\left(X,\|\cdot\|_{X}\right)$, where $X=\mathbb{R}^{\mathbb{Z}}$ and the norm in this space is monotone, i.e.

$$
\left\|\left\{a_{n}\right\}\right\|_{X} \leq\left\|\left\{b_{n}\right\}\right\|_{X} \quad \text { if }\left|a_{n}\right| \leq\left|b_{n}\right| \text { for all } n \in \mathbb{Z} .
$$

Observe that $\left\|\left\{a_{n}\right\}\right\|_{X}=\left\|\left\{\left|a_{n}\right|\right\}\right\|_{X}$ for all $\left\{a_{n}\right\} \in X$.

Remark 2.1. Some examples of these Banach spaces are the $\ell^{p}(\mathbb{Z})$ spaces, $1 \leq p<$ $\infty$, and the space where the norm is associated to some Young function. Observe that not all Banach spaces satisfy the condition 2.1 ; for example, consider $X=\mathbb{R}^{\mathbb{Z}}$ with the norm

$$
\left\|\left\{x_{n}\right\}\right\|_{X}:=\left(\left(x_{1}-x_{2}\right)^{2}+\sum_{n \neq 1} x_{n}^{2}\right)^{1 / 2} .
$$

Let $\left(\ldots, 0, x_{1}, x_{2}, 0, \ldots\right)=(\ldots, 0,1,3,0, \ldots)$ and $\left(\ldots, 0, y_{1}, y_{2}, 0, \ldots\right)=(\ldots, 0,2,3$, $0, \ldots)$. Observe that $\left|x_{n}\right| \leq\left|y_{n}\right|$ for all $n \in \mathbb{Z}$, and $\left\|\left\{x_{n}\right\}\right\|_{X}=\sqrt{13}$ and $\left\|\left\{y_{n}\right\}\right\|_{X}=$ $\sqrt{10}$. Hence, the norm is not monotone.

Remark 2.2. If $X$ is a Banach lattice, the norm is monotone by definition.

Now, we define the notion of Young function, maximal operators related to a Young function, and generalized Hörmander condition. For more details see [15].

A function $\mathcal{A}:[0, \infty) \rightarrow[0, \infty)$ is said to be a Young function if $\mathcal{A}$ is continuous, convex, non-decreasing, and satisfies $\mathcal{A}(0)=0$ and $\lim _{t \rightarrow \infty} \mathcal{A}(t)=\infty$.

The average of the Luxemburg norm of a function $f$ induced by a Young function $\mathcal{A}$ in the ball $B$ is defined by

$$
\|f\|_{\mathcal{A}, B}:=\inf \left\{\lambda>0: \frac{1}{|B|} \int_{B} \mathcal{A}\left(\frac{|f|}{\lambda}\right) \leq 1\right\} .
$$

Observe that if $\mathcal{A}(t)=t^{r}, r \geq 1$, then $\|f\|_{\mathcal{A}, B}=\left(\frac{1}{|B|} \int_{B}|f|^{r}\right)^{1 / r}$.

Each Young function $\mathcal{A}$ has an associated complementary Young function $\overline{\mathcal{A}}$ satisfying the generalized Hölder inequality

$$
\frac{1}{|B|} \int_{B}|f g| \leq 2\|f\|_{\mathcal{A}, B}\|g\|_{\overline{\mathcal{A}}, B} .
$$
then

If $\mathcal{A}, \mathcal{B}, \mathcal{C}$ are Young functions satisfying $\mathcal{A}^{-1}(t) \mathcal{B}^{-1}(t) \mathcal{C}^{-1}(t) \leq t$, for all $t \geq 1$,

$$
\|f g h\|_{L^{1}, B} \leq c\|f\|_{\mathcal{A}, B}\|g\|_{\mathcal{B}, B}\|h\|_{\mathcal{C}, B} .
$$

Given $f \in L_{\text {loc }}^{1}\left(\mathbb{R}^{n}\right)$, the maximal operator associated to the Young function $\mathcal{A}$ is defined as

$$
M_{\mathcal{A}} f(x):=\sup _{B \ni x}\|f\|_{\mathcal{A}, B}
$$


For example, if $\beta \geq 0$ and $r \geq 1, \mathcal{A}(t)=t^{r}(1+\log (t))^{\beta}$ is a Young function then $M_{\mathcal{A}}=M_{L^{r}(\log L)^{\beta}}$. If $\beta=0, \mathcal{A}(t)=t^{r}$ then $M_{\mathcal{A}}=M_{r}$, where $M_{r} f=M\left(f^{r}\right)^{1 / r}$. If $r=1$ and $\beta=k \in \mathbb{N}, M_{\mathcal{A}}=M_{L(\log L)^{k}} \approx M^{k+1}$, where $M^{k}$ is the $k$-iterated of $M$, the Hardy-Littlewood maximal.

Remark 2.3. Let us observe that when $\mathcal{D}(t)=t$, which gives $L^{1}$, then $\overline{\mathcal{D}}(t)=0$ if $t \leq 1$ and $\overline{\mathcal{D}}(t)=\infty$ otherwise. Observe that $\overline{\mathcal{D}}$ is not a Young function but one has $L^{\overline{\mathcal{D}}}=L^{\infty}$. Besides, the inverse is $\overline{\mathcal{D}}^{-1} \equiv 1$ and the generalized Hölder inequality makes sense if one of the three functions is $\overline{\mathcal{D}}$.

Once the Luxemburg average has been defined, we can introduce the notion of the generalized Hörmander condition; for this we need to introduce some notation: $|x| \sim s$ means $s<|x| \leq 2 s$, and given a Young function $\mathcal{A}$, we write

$$
\|f\|_{\mathcal{A},|x| \sim s}=\left\|f \chi_{|x| \sim s}\right\|_{\mathcal{A}, B(0,2 s)} .
$$

In 111 and 12 the following classes were introduced.

Definition A. Let $K$ be a vector-valued function, $\mathcal{A}$ be a Young function, and $k \in \mathbb{N} \cup\{0\}$. Then $K$ satisfies the $L^{\mathcal{A}, X, k}$-Hörmander condition $\left(K \in H_{\mathcal{A}, X, k}\right)$, if there exist $c_{\mathcal{A}}>1$ and $C_{\mathcal{A}}>0$ such that for all $x$ and $R>c_{\mathcal{A}}|x|$ :

$$
\sum_{m=1}^{\infty}\left(2^{m} R\right)^{n} m^{k}\|\| K(\cdot-x)-K(\cdot)\left\|_{X}\right\|_{\mathcal{A},|y| \sim 2^{m} R} \leq C_{\mathcal{A}}
$$

We say that $K \in H_{\infty, k}$ if $K$ satisfies the previous condition with $\|\cdot\|_{L^{\infty},|x| \sim 2^{m} R}$ in place of $\|\cdot\|_{\mathcal{A},|x| \sim 2^{m} R}$.

If $k=0$, we denote $H_{\mathcal{A}, X}=H_{\mathcal{A}, X, 0}$ and $H_{\infty, X}=H_{\infty, X, 0}$.

Remark 2.4. There exists a relation between the Hörmander classes, $H_{\mathcal{A}, X, k}$.

(1) $H_{\infty, X, k} \subset H_{\mathcal{A}, X, k} \subset H_{\mathcal{A}, X, k-1} \subset \cdots \subset H_{\mathcal{A}, X, 0}=H_{\mathcal{A}, X} \subset H_{1, X}$, for $k \in \mathbb{N}$.

(2) If $\mathcal{A}$ and $\mathcal{B}$ are Young functions such that $\mathcal{A}(t) \leq c \mathcal{B}(t)$ for $t>t_{0}$, some $t_{0}>0$, then

$$
H_{\infty, X, k} \subset H_{\mathcal{B}, X, k} \subset H_{\mathcal{A}, X, k} \subset H_{1, X, k} \subset H_{1, X} .
$$

(3) In the particular case of $\mathcal{A}(t)=t^{r}, 1 \leq r<\infty$, denoting $H_{r, X}=H_{\mathcal{A}, X}$, it follows that

$H_{\infty, X, k} \subset H_{r_{2}, X, k} \subset H_{r_{1}, X, k} \subset H_{1, X, k} \subset H_{1, X}$, for all $1<r_{1}<r_{2}<\infty$.

Next, we define the notions of singular integral operator and its commutator in the vector-valued sense.

Definition 2.5. Consider a vector-valued function $K, K(y)=\left\{K_{l}(y)\right\}_{l \in \mathbb{Z}}$, with $K_{l} \in L_{\text {loc }}^{1}\left(\mathbb{R}^{n} \backslash\{0\}\right)$. Let

$$
\begin{aligned}
T f(x) & :=\text { p.v. } \int_{\mathbb{R}^{n}} K(x-y) f(y) d y=\left\{\left(K_{l} * f\right)(x)\right\}_{l \in \mathbb{Z}} \\
& =\left\{\text { p.v. } \int_{\mathbb{R}^{n}} K_{l}(x-y) f(y) d y\right\}_{l \in \mathbb{Z}} .
\end{aligned}
$$


The operator $T$ will be a singular integral operator if it is strong $\left(p_{0}, p_{0}\right)$, for some $p_{0}>1$, and the kernel $K=\left\{K_{l}\right\}_{l \in \mathbb{Z}} \in H_{1, X}$.

Remark 2.6. The operator $T$ is strong $\left(p_{0}, p_{0}\right)$ in the sense of Bochner-Lebesgue spaces. Given a Banach space $X, L_{X}^{p}\left(\mathbb{R}^{n}\right)$ is called Bochner-Lebesgue space with the norm $\left(\int_{\mathbb{R}^{n}}\|f(x)\|_{X}^{p} d x\right)^{1 / p}$.

Remark 2.7. Since $K=\left\{K_{l}\right\}_{l \in \mathbb{Z}} \in H_{1, X}, T$ is of weak type $(1,1)$ and satisfies Kolmogorov's inequality,

$$
\left(\frac{1}{|B|} \int_{B}\|T f\|_{\mathbf{x}}^{\varepsilon}\right)^{\frac{1}{\varepsilon}} \leq c \frac{1}{|\widehat{B}|} \int_{\widehat{B}}|f|
$$

where $0<\varepsilon<1$ and $\operatorname{supp}(f)=\widehat{B} \subset B$.

Let us recall the BMO space and the sharp maximal function. If $f \in L_{\text {loc }}^{1}\left(\mathbb{R}^{n}\right)$ define

$$
M^{\sharp} f(x)=\sup _{B \ni x} \frac{1}{|B|} \int_{B}\left|f-\frac{1}{|B|} \int_{B} f\right| .
$$

A locally integrable function $f$ has bounded mean oscillation $(f \in \mathrm{BMO})$ if $M^{\sharp} f \in L^{\infty}$ and the norm $\|f\|_{\mathrm{BMO}}=\left\|M^{\sharp} f\right\|_{\infty}$.

Observe that the BMO norm is equivalent to

$$
\|f\|_{\mathrm{BMO}}=\left\|M^{\sharp} f\right\|_{\infty} \sim \operatorname{supinf}_{B} \frac{1}{a \in \mathbb{C}|B|} \int_{B}|f(x)-a| d x .
$$

Remark 2.8. The following are some properties of BMO:

Given $b \in \mathrm{BMO}$, a ball $B, k \in \mathbb{N} \cup\{0\}, \mathcal{A}(t)=\exp \left(t^{1 / k}\right)$ and $q>0$, by John-Nirenberg's Theorem we have

$$
\left\|\left(b-b_{B}\right)^{k}\right\|_{L^{q}, B} \leq\left\|\left(b-b_{B}\right)^{k}\right\|_{\mathcal{A}, B}=\left\|b-b_{B}\right\|_{\exp L, B}^{k} \leq C\|b\|_{\mathrm{BMO}} .
$$

On the other hand, for any $j \in \mathbb{N}$ and $b \in \mathrm{BMO}$, we have

$$
\left|b_{B}-b_{2^{j} B}\right| \leq \sum_{m=1}^{j}\left|b_{2^{m-1} B}-b_{2^{m} B}\right| \leq 2^{n} \sum_{m=1}^{j}\left\|b-b_{2^{m} B}\right\|_{L^{1}, 2^{m} B} \leq 2^{n} j\|b\|_{\text {BMO }} .
$$

Definition 2.9. Given $T$ a singular integral operator and $b \in \mathrm{BMO}$, the $k$-th order commutator of $T, k \in \mathbb{N} \cup\{0\}$, is defined by:

$$
\begin{aligned}
T_{b}^{k} f(x) & :=\text { p.v. } \int_{\mathbb{R}^{n}}(b(x)-b(y))^{k} K(x-y) f(y) d y \\
& =\left\{\text { p.v. } \int_{\mathbb{R}^{n}}(b(x)-b(y))^{k} K_{l}(x-y) f(y) d y\right\}_{l \in \mathbb{Z}} .
\end{aligned}
$$

Note that for $k=0, T_{b}^{k}=T$ and observe that $T_{b}^{k}=\left[b, T_{b}^{k-1}\right], k \in \mathbb{N}$.

Remark 2.10. $T_{b}^{k} f(x)=\left[b, T_{b}^{k-1}\right](f)(x):=b(x) T_{b}^{k-1}(f)(x)-T_{b}^{k-1}(b f)(x)$. 
We will consider weights in the Muckenhoupt classes $A_{p}, 1 \leq p \leq \infty$. Let $w$ be a non-negative locally integrable function. We say that $w \in A_{p}$ if there exists $C_{p}<\infty$ such that for any ball $B \subset \mathbb{R}^{n}$,

$$
\left(\frac{1}{|B|} \int_{B} w\right)\left(\frac{1}{|B|} \int_{B} w^{-\frac{1}{p-1}}\right)^{p-1}<C_{p}
$$

when $1<p<\infty$, and for $p=1$,

$$
M w(x) \leq C_{1} w(x), \quad \text { for a.e. } x \in \mathbb{R}^{n} .
$$

Finally we set $A_{\infty}=\cup_{1<p} A_{p}$. It is well known that the Muckenhoupt classes characterize the boundedness of the Hardy-Littlewood maximal function on weighted $L^{p}$-Lebesgue spaces. Namely, $w \in A_{p}, 1<p \leq \infty$, if and only if $M$ is bounded on $L^{p}(w)$; and $w \in A_{1}$ if and only if $M$ maps $L^{1}(w)$ into $L^{1, \infty}(w)$.

In [12] and [11] the following results were proved.

Theorem B ([12]). Let $K$ be a vector-valued function that satisfies the $L^{\mathcal{A}, X}$ Hörmander condition and let $T$ be the operator associated to $K$. Suppose $T$ is bounded in some $L^{p_{0}}, 1<p_{0}<\infty$. Then, for any $0<p<\infty$ and $w \in A_{\infty}$, there exists $C$ such that

$$
\int_{\mathbb{R}^{n}}\|T f\|_{\mathbf{x}}^{p} w \leq C \int_{\mathbb{R}^{n}}\left(M_{\overline{\mathcal{A}}} f\right)^{p} w
$$

for any $f \in C_{c}^{\infty}$ and whenever the left-hand side is finite.

For commutators of the operator $T$, there is the following result:

Theorem $\mathbf{C}$ ([11]). Let $b \in \mathrm{BMO}$ and $k \in \mathbb{N} \cup\{0\}$. Let $\mathcal{A}, \mathcal{B}$ be Young functions such that $\overline{\mathcal{A}}^{-1}(t) \mathcal{B}^{-1}(t) \overline{\mathcal{C}}_{k}^{-1}(t) \leq t$, with $\overline{\mathcal{C}}_{k}(t)=\exp \left(t^{1 / k}\right)$ for $t \geq 1$ if $k \in \mathbb{N}$ and $\overline{\mathcal{C}}_{k}^{-1} \equiv 1$ if $k=0$. If $T$ is a singular integral operator with kernel $K \in$ $H_{\mathcal{B}, X} \cap H_{\mathcal{A}, X, k}$, then for any $0<p<\infty$ and $w \in A_{\infty}$,

$$
\int_{\mathbb{R}^{n}}\left\|T_{b}^{k} f\right\|_{X}^{p} w \leq C \int_{\mathbb{R}^{n}}\left(M_{\overline{\mathcal{A}}} f\right)^{p} w, \quad f \in L_{c}^{\infty},
$$

whenever the left-hand side is finite. Furthermore, if $\overline{\mathcal{A}}$ is sub-multiplicative, then for all $w \in A_{\infty}$ and $\lambda>0$,

$$
w\left\{x \in \mathbb{R}^{n}:\left|T_{b}^{k} f(x)\right|>\lambda\right\} \leq c \int_{\mathbb{R}^{n}} \overline{\mathcal{A}}\left(\frac{\|b\|_{\mathrm{BMO}}^{k}|f(x)|}{\lambda}\right) M w(x) d x .
$$

\section{MAin RESUlts}

In this section we will state a new condition, weaker than the generalized Hörmander condition (Definition A). The previous Theorems B and C still remain true using this new condition.

Definition 3.1. Let $K$ be a vector-valued function, $\mathcal{A}$ be a Young function and $k \in$ $\mathbb{N} \cup\{0\}$. The function $K$ satisfies the $L_{\dagger}^{\mathcal{A}, X, k}$-Hörmander condition $\left(K \in H_{\mathcal{A}, X, k}^{\dagger}\right)$, 
if there exist $c_{\mathcal{A}}>1$ and $C_{\mathcal{A}}>0$ such that for all $x$ and $R>c_{\mathcal{A}}|x|$,

$$
\left\|\left\{\sum_{m=1}^{\infty}\left(2^{m} R\right)^{n} m^{k}\left\|K_{l}(\cdot-x)-K_{l}(\cdot)\right\|_{\mathcal{A},|y| \sim 2^{m} R}\right\}_{l \in \mathbb{Z}}\right\|_{X} \leq C_{\mathcal{A}} .
$$

We say that $K \in H_{\infty, k}^{\dagger}$ if $K$ satisfies the previous condition with $\|\cdot\|_{L^{\infty},|x| \sim 2^{m} R}$ in place of $\|\cdot\|_{\mathcal{A},|x| \sim 2^{m} R}$.

If $k=0$, we denote $H_{\mathcal{A}, X}^{\dagger}=H_{\mathcal{A}, X, 0}^{\dagger}$ and $H_{\infty, X}^{\dagger}=H_{\infty, X, 0}^{\dagger}$.

Remark 3.2. The classes $H_{\mathcal{A}, X, k}^{\dagger}$ satisfy the same inclusion of the classes $H_{\mathcal{A}, X, k}$ (see Remark 2.4), and the relation between these classes is the following:

$$
H_{\mathcal{A}, X, k} \subsetneq H_{\mathcal{A}, X, k}^{\dagger}
$$

In section 4, we give an explicit example of a kernel $K$ such that $K \in H_{\mathcal{A}, X, k}^{\dagger}$ and $K \notin H_{\mathcal{A}, X, k}$ (see Proposition $\mathrm{F}$ and Corollary 4.5 .

Using Definition 3.1. the previous theorems are written as follows, for the case $k=0$ :

Theorem 3.3. Let $T$ be a vector-valued singular integral operator with kernel $K \in H_{\mathcal{A}, X}^{\dagger}$. Then, for any $0<p<\infty$ and $w \in A_{\infty}$, there exists $C$ such that

$$
\int_{\mathbb{R}^{n}}\|T f\|_{X}^{p} w \leq C \int_{\mathbb{R}^{n}}\left(M_{\overline{\mathcal{A}}} f\right)^{p} w, \quad f \in L_{c}^{\infty}\left(\mathbb{R}^{n}\right)
$$

whenever the left-hand side is finite.

And for the case $k \in \mathbb{N}$ :

Theorem 3.4. Let $b \in \mathrm{BMO}$ and $k \in \mathbb{N}$. Let $\mathcal{A}, \mathcal{B}$ be Young functions such that $\overline{\mathcal{A}}^{-1}(t) \mathcal{B}^{-1}(t) \overline{\mathcal{C}}_{k}^{-1}(t) \leq t$, with $\overline{\mathcal{C}}_{k}(t)=\exp \left(t^{1 / k}\right)$ for $t \geq 1$. If $T$ is a vector-valued singular integral operator with kernel $K \in H_{\mathcal{B}, X}^{\dagger} \cap H_{\mathcal{A}, X, k}^{\dagger}$, then for any $0<p<\infty$ and $w \in A_{\infty}$, there exists $C$ such that

$$
\int_{\mathbb{R}^{n}}\left\|T_{b}^{k} f\right\|_{X}^{p} w \leq C \int_{\mathbb{R}^{n}}\left(M_{\overline{\mathcal{A}}} f\right)^{p} w, \quad f \in L_{c}^{\infty}\left(\mathbb{R}^{n}\right),
$$

whenever the left-hand side is finite.

Furthermore, if $\overline{\mathcal{A}}$ is sub-multiplicative, then for all $w \in A_{\infty}$ and $\lambda>0$,

$$
w\left\{x \in \mathbb{R}^{n}:\left|T_{b}^{k} f(x)\right|>\lambda\right\} \leq c \int_{\mathbb{R}^{n}} \overline{\mathcal{A}}\left(\frac{\|b\|_{\mathrm{BMO}}^{k}|f(x)|}{\lambda}\right) M w(x) d x .
$$

Remark 3.5. These theorems are more general than Theorem $\mathrm{B}$ and $\mathrm{C}$ since there exists a singular integral operator whose kernel $K \in H_{\mathcal{A}, X, k}^{\dagger}$ and $K \notin H_{\mathcal{A}, X, k}$ for some appropriate Young function $\mathcal{A}$.

Let $\mathcal{A}(t)=\exp \left(t^{\frac{1}{1+k}}\right)-1$ and $\overline{\mathcal{C}}_{k}(t)=\exp \left(t^{1 / k}\right)$. If $\mathcal{B}(t)=\exp (t)-1$ then $\overline{\mathcal{A}}^{-1}(t) \mathcal{B}^{-1}(t) \overline{\mathcal{C}}_{k}^{-1}(t) \leq t$. Thus, if $K \in H_{\mathcal{A}, X, k}^{\dagger}$ then $K \in H_{\mathcal{B}, X}^{\dagger}$. In this case Theorems 3.3 and 3.4 can be written as follows. 
Theorem 3.6. Let $b \in \mathrm{BMO}$ and $k \in \mathbb{N} \cup\{0\}$. Let $\mathcal{A}(t)=\exp \left(t^{\frac{1}{1+k}}\right)-1$. If $T$ is a vector-valued singular integral operator with kernel $K \in H_{\mathcal{A}, X, k}^{\dagger}$, then for any $0<p<\infty$ and $w \in A_{\infty}$, there exists $C$ such that

$$
\int_{\mathbb{R}^{n}}\left\|T_{b}^{k} f\right\|_{X}^{p} w \leq C \int_{\mathbb{R}^{n}}\left(M_{\overline{\mathcal{A}}} f\right)^{p} w \leq C \int_{\mathbb{R}^{n}}\left(M^{k+2} f\right)^{p} w, \quad f \in L_{c}^{\infty}\left(\mathbb{R}^{n}\right),
$$

whenever the left-hand side is finite.

Furthermore, for all $w \in A_{\infty}$ and $\lambda>0$,

$$
w\left\{x \in \mathbb{R}^{n}:\left|T_{b}^{k} f(x)\right|>\lambda\right\} \leq c \int_{\mathbb{R}^{n}} \overline{\mathcal{A}}\left(\frac{\|b\|_{\mathrm{BMO}}^{k}|f(x)|}{\lambda}\right) M w(x) d x,
$$

where $\overline{\mathcal{A}}(t)=t(1+\log (t))^{k+1}$.

\section{Applichtions and generalization}

Now, we define the vector-valued singular integral operator, $\tilde{T}$, and its commutator, that will be an example of our results.

Definition 4.1. Let $f$ be a locally integrable function in $\mathbb{R}$. Let $\tilde{T}$ be defined as:

$$
\begin{aligned}
\tilde{T} f(x) & :=\left\{\int_{\mathbb{R}}\left(\frac{1}{2^{l+1}} \chi_{\left(-2^{l}, 2^{l}\right)}(x-y)-\frac{1}{2^{l}} \chi_{\left(-2^{l-1}, 2^{l-1}\right)}(x-y)\right) f(y) d y\right\}_{l \in \mathbb{Z}} \\
& =\int_{\mathbb{R}} K(x-y) f(y) d y,
\end{aligned}
$$

where $K$ is

$$
K(z)=\left\{K_{l}(z)\right\}_{l \in \mathbb{Z}}=\left\{\frac{1}{2^{l+1}} \chi_{\left(-2^{l}, 2^{l}\right)}(z)-\frac{1}{2^{l}} \chi_{\left(-2^{l-1}, 2^{l-1}\right)}(z)\right\}_{l \in \mathbb{Z}}
$$

For this operator $\tilde{T}$, the Banach space $\left(X,\|\cdot\|_{X}\right)$ will be $\left(\ell^{2}(\mathbb{Z}),\|\cdot\|_{\ell^{2}}\right)$.

Definition 4.2. Let $f$ be a measurable function in $\mathbb{R}, k \in \mathbb{N} \cup\{0\}$, and $b \in$ BMO. The $k$-th order commutator is defined as

$$
S_{b}^{k} f(x):=\left\|\tilde{T}_{b}^{k} f(x)\right\|_{\ell^{2}}
$$

where $\tilde{T}_{b}^{k}$ is the $k$-th order commutator of $\tilde{T}$. The $S_{b}^{k}$ is called the $k$-th commutator of the square operator.

In [11] and [17] the authors studied the kernel of the square operator for the one-sided case. The following results are for the two-sided case and the proofs are analogous to the one-sided case. 
Proposition D ([17]). Let $x_{0} \in \mathbb{R}$ and $i, j \in \mathbb{Z}, i<j$. Let $x, y \in \mathbb{R}$ such that $\left|x-x_{0}\right|<2^{i}, y \in\left(x_{0}-2^{j+1}, x_{0}-2^{j}\right)$ or $y \in\left(x_{0}+2^{j}, x_{0}+2^{j+1}\right)$. Then

$$
\begin{aligned}
& \left|K_{l}(y-x)-K_{l}\left(y-x_{0}\right)\right| \\
& \quad= \begin{cases}\frac{1}{2^{j+1}} \chi_{\left(x-2^{j}, x_{0}-2^{j}\right) \cup\left(x_{0}+2^{j}, x+2^{j}\right)}(y) & \text { if } l=j, \\
\frac{1}{2^{j+2}} \chi_{\left(x_{0}-2^{j+1}, x-2^{j+1}\right) \cup\left(x+2^{j+1}, x_{0}+2^{j+1}\right)}(y) & \\
+\frac{1}{2^{j+1}} \chi_{\left(x-2^{j}, x_{0}-2^{j}\right) \cup\left(x_{0}+2^{j}, x+2^{j}\right)}(y) & \text { if } l=j+1, \\
\frac{1}{2^{j+2}} \chi_{\left(x_{0}-2^{j+1}, x-2^{j+1}\right) \cup\left(x+2^{j+1}, x_{0}+2^{j+1}\right)}(y) & \text { if } l=j+2, \\
0 & \text { if } l \notin\{j, j+1, j+2\} .\end{cases}
\end{aligned}
$$

In [12, using Proposition $\mathrm{D}$ the authors proved the following results.

Proposition E ([12]). The kernel $K \notin H_{\infty}, \ell^{2}$.

Remark 4.3. As $K \notin H_{\infty, \ell^{2}, k}$ we can not use Theorem 3.4 to conclude that

$$
\int_{\mathbb{R}}\left|S_{b}^{k} f(x)\right|^{p} w(x) d x=\int_{\mathbb{R}}\left\|\tilde{T}_{b}^{k} f(x)\right\|_{\ell^{2}}^{p} w(x) d x \leq C \int_{\mathbb{R}}\left|M^{k+1} f(x)\right|^{p} w(x) d x .
$$

This inequality is still an open problem.

Proposition F $([11])$. Let $\mathcal{A}_{\varepsilon}(t)=\exp \left(t^{\frac{1}{1+k+\varepsilon}}\right)-1, \varepsilon \geq 0$, and $k \in \mathbb{N} \cup\{0\}$. Then, $K \in H_{\mathcal{A}_{\varepsilon}, \ell^{2}, k}$ for all $\varepsilon>0$, and $K \notin H_{\mathcal{A}_{0}, \ell^{2}, k}$.

In 11] and [12, as an application of Theorems B and $\mathrm{C}$ the authors obtained the following result.

Theorem G $([11,12])$. Let $b \in \mathrm{BMO}$ and $k \in \mathbb{N} \cup\{0\}$. Let $S_{b}^{k}$ be the $k$-th order commutator of the square operator. Then for any $0<p<\infty$ and $w \in A_{\infty}$, there exists $C$ such that

$$
\int_{\mathbb{R}}\left(S_{b}^{k} f(x)\right)^{p} w(x) d x=\int_{\mathbb{R}}\left(\left\|\tilde{T}_{b}^{k} f(x)\right\|_{\ell^{2}}\right)^{p} w(x) d x \leq C \int_{\mathbb{R}}\left(M^{k+3} f(x)\right)^{p} w(x) d x,
$$

whenever the left-hand side is finite.

For the case of the kernel of the square operator we obtain:

Proposition 4.4. Let $k \in \mathbb{N} \cup\{0\}$ and $\mathcal{A}$ be a Young function. Then,

$$
K \in H_{\mathcal{A}, \ell^{2}, k}^{\dagger} \Leftrightarrow\left\|\left\{\frac{m^{k}}{\mathcal{A}^{-1}\left(2^{m} 8\right)}\right\}_{m \in \mathbb{Z}}\right\|_{\ell^{2}}<\infty .
$$

Corollary 4.5. Let $\mathcal{A}(t)=\exp \left(t^{\frac{1}{1+k}}\right)-1$. Then the kernel $K \in H_{\mathcal{A}, \ell^{2}, k}^{\dagger}$ for any $k \in \mathbb{N} \cup\{0\}$.

Corollary 4.5 tells us that the kernel of the square operator satisfies the hypotheses of Theorems 3.3 and 3.4 (see Theorem 3.6 and we obtain a new proof of the following result. 
Theorem $\mathbf{H}([13])$. Let $b \in \mathrm{BMO}$ and $k \in \mathbb{N} \cup\{0\}$. Let $S_{b}^{k}$ be the $k$-th order commutator of the square operator. Then, for any $0<p<\infty$ and $w \in A_{\infty}$, there exists $C$ such that

$$
\int_{\mathbb{R}}\left(S_{b}^{k} f(x)\right)^{p} w(x) d x \leq C \int_{\mathbb{R}}\left(M^{k+2} f(x)\right)^{p} w(x) d x,
$$

whenever the left-hand side is finite.

4.1. Generalization of the square operator. In this subsection we will build a family of operators and we will prove that they satisfy Proposition 4.4. These operators are a generalization of the square operator.

Let $X$ be a Banach space with a monotone norm, see (2.1). We define $S_{X} f(x):=$ $\|\tilde{T} f(x)\|_{X}$, where $\tilde{T}$ was defined in Definition 4.1 Observe that if $X=\ell^{2}$ then $S_{X}=S$, the square operator.

We can generalize Proposition 4.4 and Corollary 4.5 , replacing the $\ell^{2}$-norm by the $X$-norm. In this case, Proposition 4.4 states that for all $k \geq 0$ and $\mathcal{A}$ a Young function,

$$
K \in H_{\mathcal{A}, X, k}^{\dagger} \Leftrightarrow\left\|\left\{\frac{m^{k}}{\mathcal{A}^{-1}\left(2^{m} 8\right)}\right\}_{m \in \mathbb{Z}}\right\|_{X}<\infty .
$$

Also, Corollary 4.5 can be rewritten in this way: If $\mathcal{A}(t)=\exp \left(t^{\frac{1}{1+k}}\right)-1$ and $k \in \mathbb{N} \cup\{0\}$, then $K \in H_{\mathcal{A}, X, k}^{\dagger}$. have

Observe that if $k \in \mathbb{N} \cup\{0\}$ and $\mathcal{A}(t)=\exp \left(t^{\frac{1}{1+k}}\right)-1$, by Proposition 4.4 we

$$
K \in H_{\mathcal{A}, X, k}^{\dagger} \Leftrightarrow\left\|\left\{\frac{1}{m}\right\}_{m \in(\mathbb{Z}-\{0\})}\right\|_{X}=C_{\mathcal{A}, X}<\infty .
$$

Applying Theorem 3.6, we obtain

$$
\begin{aligned}
\int_{\mathbb{R}^{n}}\left|S_{X, b}^{k} f(x)\right|^{p} w(x) d x & =\int_{\mathbb{R}^{n}}\left\|\tilde{T}_{b}^{k} f(x)\right\|_{X}^{p} w(x) d x \\
& \leqslant c \int_{\mathbb{R}^{n}}\left(M_{\overline{\mathcal{A}}} f(x)\right)^{p} w(x) d x \leqslant c \int_{\mathbb{R}^{n}}\left(M^{k+2} f(x)\right)^{p} w(x) d x,
\end{aligned}
$$

whenever the left-hand side is finite.

Remark 4.6. Examples of the Banach spaces $X$ are the $\ell^{p}$ spaces with $1 \leq p \leq \infty$. Observe that for $p=2$ condition 4.2 holds, but it is easy to see that it does not hold for $p=1$. One open question is: Does there exist a Young function $\mathcal{A}$ such that the condition 4.1 is finite for $X=\ell^{1}$ ? For example, does there exist a Young function such that the condition 4.2 is replaced by $\left\|\left\{\frac{1}{m^{2}}\right\}_{m \in(\mathbb{Z}-\{0\})}\right\|_{\ell^{1}}$ ?

An interesting example is: given a Young function $\mathcal{E}$, we denote $X_{\mathcal{E}}=\left(\mathbb{R}^{\mathbb{Z}},\|\cdot\|_{\mathcal{E}}\right)$, the Banach space with

$$
\left\|\left\{a_{n}\right\}\right\|_{\mathcal{E}}=\inf \left\{\lambda>0: \sum_{n \in \mathbb{Z}} \mathcal{E}\left(\frac{\left|a_{n}\right|}{\lambda}\right) \leq 1\right\} .
$$


Now we give an example of a family of Young functions for which condition 4.2 holds. Let us consider, for $t \geq 0$, the Young function $\mathcal{E}(t)=t^{r}(\log (t+1))^{\beta}$, where $\beta \geq 0$ and $r \geq 1$. For this it is enough to prove that there exists $0<\lambda<\infty$ such that $\lambda \in G$, where

$$
G:=\left\{\lambda>0: \sum_{m \in \mathbb{Z}-\{0\}} \mathcal{E}\left(\frac{1 / m}{\lambda}\right) \leq 1\right\} .
$$

Let $\lambda>1$,

$$
\begin{aligned}
\sum_{m \in \mathbb{Z}-\{0\}} \mathcal{E}\left(\frac{1 / m}{\lambda}\right) & =\sum_{m \in(\mathbb{Z}-\{0\})} \log \left(\frac{1}{|m| \lambda}+1\right)^{\beta} \frac{1}{(\lambda m)^{r}} \\
& \leq \log \left(\frac{1}{\lambda}+1\right)^{\beta} \frac{1}{\lambda^{r}} \sum_{m \in(\mathbb{Z}-\{0\})} \frac{1}{m^{r}} \\
& \leq \log (2)^{\beta} \frac{1}{\lambda^{r}} c .
\end{aligned}
$$

Observe that $\log (2)^{\beta} \frac{1}{\lambda^{r}} c \leq 1$ if and only if $c \log (2)^{\beta} \leq \lambda^{r}$. In particular, $\lambda_{0}:=$ $\left(\log (2)^{\beta} C+1\right)^{1 / r}$ satisfies this inequality, i.e., $\lambda_{0} \in G$. Thus, we have that 4.2 is true.

4.2. Proof of Proposition 4.4 and Corollary 4.5. In this subsection we proceed to study the applications. Let $K$ be the kernel of the square operator, defined above.

Definition. We define the sets

$$
\begin{aligned}
& -F_{m}^{-}:=\left(x-2^{m+i},-2^{m+i}\right) \quad F_{m}^{-}:=\left(x+2^{m+i}, 2^{m+i}\right) \\
& -F_{m}^{+}:=\left(-2^{m+i}, x-2^{m+i}\right) \quad F_{m}^{+}:=\left(2^{m+i}, x+2^{m+i}\right) \\
& -F_{m}:=\left\{\begin{array}{ll}
-F_{m}^{-} & \text {if } x<0, \\
-F_{m}^{+} & \text {if } x>0,
\end{array} \quad F_{m}:= \begin{cases}F_{m}^{-} & \text {if } x<0, \\
F_{m}^{+} & \text {if } x>0 .\end{cases} \right.
\end{aligned}
$$

Observe that if $|x|<2^{i},\left[-F_{m} \cup F_{m}\right] \cap\left[-F_{m-1} \cup F_{m-1}\right]=\emptyset$, for all $m \in \mathbb{Z}$.

Proof of Proposition 4.4. Recall that $K \in H_{\mathcal{A}, \ell^{2}, k}^{\dagger}$ if there exist $c_{\mathcal{A}}>1$ and $C_{\mathcal{A}}>0$ such that for each $x$ and $R>c_{\mathcal{A}}|x|$,

$$
\left\|\left\{\sum_{m=1}^{\infty}\left(2^{m} R\right)^{n} m^{k}\left\|\left(K_{l}(\cdot-x)-K_{l}(\cdot)\right) \chi_{|y| \sim 2^{m} R}(\cdot)\right\|_{\mathcal{A}, B\left(0,2^{m+1} R\right)}\right\}_{l \in \mathbb{Z}}\right\|_{X} \leq C_{\mathcal{A}} .
$$

Let us prove that

$$
\left\|\left\{\frac{m^{k}}{\mathcal{A}^{-1}\left(2^{m} 8\right)}\right\}_{m \in \mathbb{Z}}\right\|_{\ell^{2}}<\infty \Rightarrow K \in H_{\mathcal{A}, \ell^{2}, k}^{\dagger} .
$$


If $x=0,\left(K_{l}(\cdot-x)-K_{l}(\cdot)\right)=0$ for all $l \in \mathbb{Z}$, then the condition $(4.3)$ is trivial. Let $x \neq 0$. Let $R=2^{i}, i \in \mathbb{Z}, x$ such that $|x|<2^{i}, I_{m}:=\left(-2^{m+i}, 2^{m+i}\right)$ and $-F_{m}$ and $F_{m}$ as above. For $l \in \mathbb{Z}$, using Proposition $\mathrm{D}$ we obtain

$$
\begin{aligned}
\sum_{m=1}^{\infty} 2^{m+i} m^{k} \| & \left(K_{l}(\cdot-x)-K_{l}(\cdot)\right) \chi_{|y| \sim 2^{m+i}} \|_{\mathcal{A}, I_{m+1}} \\
= & 2^{l+i} l^{k}\left\|\frac{1}{2^{l+i+1}} \chi_{-F_{l} \cup F_{l}}\right\|_{\mathcal{A}, I_{l+1}} \\
& +2^{l-1+i}(l-1)^{k}\left\|\frac{1}{2^{l+i+1}} \chi_{-F_{l} \cup F_{l}}+\frac{1}{2^{l+i}} \chi_{-F_{l-1} \cup F_{l-1}}\right\|_{\mathcal{A}, I_{l}} \\
& +2^{l-2+i}(l-2)^{k}\left\|\frac{1}{2^{l}+i} \chi_{-F_{l-1} \cup F_{l-1}}\right\|_{\mathcal{A}, I_{l-1}} \\
\leq & 2^{l+i} l^{k}\left\|\frac{1}{2^{l+i+1}} \chi_{-F_{l} \cup F_{l}}\right\|\left\|_{\mathcal{A}, I_{l+1}}+2^{l-1+i}(l-1)^{k}\right\| \frac{1}{2^{l+i+1}} \chi_{-F_{l} \cup F_{l}} \|_{\mathcal{A}, I_{l}} \\
& +2^{l-1+i}(l-1)^{k}\left\|\frac{1}{2^{l+i}} \chi_{-F_{l-1} \cup F_{l-1}}\right\|_{\mathcal{A}, I_{l}} \\
& +2^{l-2+i}(l-2)^{k}\left\|\frac{1}{2^{l+i}} \chi_{-F_{l-1} \cup F_{l-1}}\right\|_{\mathcal{A}, I_{l-1}} .
\end{aligned}
$$

Using that

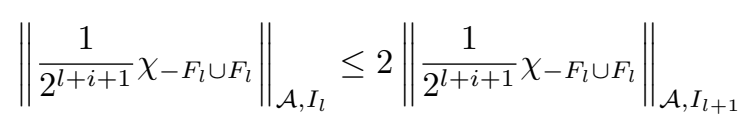

and

$$
\left\|\frac{1}{2^{l+i}} \chi_{-F_{l-1} \cup F_{l-1}}\right\|_{\mathcal{A}, I_{l-1}} \leq 2\left\|\frac{1}{2^{l+i}} \chi_{-F_{l-1} \cup F_{l-1}}\right\|_{\mathcal{A}, I_{l}}
$$

we get

$$
\begin{aligned}
& \sum_{m=1}^{\infty} 2^{m+i} m^{k}\left\|\left(K_{l}(\cdot-x)-K_{l}(\cdot)\right) \chi_{|y| \sim 2^{m+i}}\right\|_{\mathcal{A}, I_{m+1}} \\
& \quad \leq 2.2^{l+i} l^{k}\left\|\frac{1}{2^{l+i+1}} \chi_{-F_{l} \cup F_{l}}\right\|_{\mathcal{A}, I_{l+1}}+2.2^{l-1+i}(l-1)^{k}\left\|\frac{1}{2^{l+i}} \chi_{-F_{l-1} \cup F_{l-1}}\right\|_{\mathcal{A}, I_{l}} \\
& \quad=\frac{l^{k}}{\mathcal{A}^{-1}\left(\frac{2^{l+i+2}}{2|x|}\right)}+\frac{(l-1)^{k}}{\mathcal{A}^{-1}\left(\frac{2^{l+i+1}}{2|x|}\right)} \\
& \quad \leq \frac{2 l^{k}}{\mathcal{A}^{-1}\left(\frac{2^{l+i+1}}{|x|}\right)},
\end{aligned}
$$

where the last inequality holds due to $\mathcal{A}^{-1}$ being monotone. 
Then, for all $|x|<2^{i}$, we obtain

$$
\begin{gathered}
\left\|\left\{\sum_{m=1}^{\infty} 2^{m+i} m^{k}\left\|\left(K_{l}(\cdot-x)-K_{l}(\cdot)\right) \chi_{|y| \sim 2^{m+i}}\right\|_{\mathcal{A}, t_{m+1}}\right\}_{l \in \mathbb{Z}}\right\|_{\ell^{2}} \\
\leq\left\|\left\{\frac{2 l^{k}}{\mathcal{A}^{-1}\left(\frac{2^{l+i+1}}{|x|}\right)}\right\}_{l \in \mathbb{Z}}\right\|_{\ell^{2}} .
\end{gathered}
$$

In particular, the last inequality holds for all $|x|<\frac{2^{i}}{4}$. As $|x|<\frac{2^{i}}{4}$, we have $\frac{2^{l+i+1}}{|x|}>2^{l} 8$,

$$
\begin{aligned}
\|\left\{\sum_{m=1}^{\infty} 2^{m+i} m^{k} \|\right. & \left.\left(K_{l}(\cdot-x)-K_{l}(\cdot)\right) \chi_{|y| \sim 2^{m+i}} \|_{\mathcal{A}, B_{m+1}}\right\}_{l \in \mathbb{Z}} \|_{\ell^{2}} \\
\leq & \left\|\left\{\frac{2 l^{k}}{\mathcal{A}^{-1}\left(\frac{2^{l+i+1}}{|x|}\right)}\right\}_{l \in \mathbb{Z}}\right\|_{\ell^{2}} \\
& \leq\left\|\left\{\frac{2 l^{k}}{\mathcal{A}^{-1}\left(2^{l} 8\right)}\right\}_{l \in \mathbb{Z}}\right\|_{\ell^{2}}=2\left\|\left\{\frac{l^{k}}{\mathcal{A}^{-1}\left(2^{l} 8\right)}\right\}_{l \in \mathbb{Z}}\right\|_{\ell^{2}} .
\end{aligned}
$$

Then, by hypothesis, we obtain $K \in H_{\mathcal{A}, \ell^{2}, k}^{\dagger}$.

Now let us prove that $K \in H_{\mathcal{A}, \ell^{2}, k}^{\dagger} \Rightarrow\left\|\left\{\frac{m^{k}}{\mathcal{A}^{-1}\left(2^{m} 8\right)}\right\}_{m \in \mathbb{Z}}\right\|_{\ell^{2}}<\infty$. By hypothesis, there exist $c_{\mathcal{A}}>1$ and $C_{\mathcal{A}}>0$ such that for all $R \in \mathbb{R}$ and for all $x,|x| c_{\mathcal{A}}<2^{i}$, then

$$
\left\|\left\{\sum_{m=1}^{\infty} 2^{m} R m^{k}\left\|\left(K_{l}(\cdot-x)-K_{l}(\cdot)\right) \chi_{|y| \sim 2^{m} R}\right\|_{\mathcal{A}, B_{m+1}}\right\}_{l \in \mathbb{Z}}\right\|_{\ell^{2}} \leq C_{\mathcal{A}} .
$$

Let $i \in \mathbb{Z}$. If $R=2^{i}$, then $|x|<2^{i}$. Thus, using Proposition $\mathrm{D}$ we get

$$
\begin{aligned}
\|\left\{\sum_{m=1}^{\infty} 2^{m+i} m^{k} \|\left(K_{l}(\cdot-x)\right.\right. & \left.\left.-K_{l}(\cdot)\right) \chi_{|y| \sim 2^{m+i}} \|_{\mathcal{A}, B_{m+1}}\right\}_{l \in \mathbb{Z}} \|_{\ell^{2}} \\
& \geq\left\|\left\{2^{l+i} l^{k}\left\|\frac{1}{2^{l+i+1}} \chi_{-F_{l} \cup F_{l}}\right\|_{\mathcal{A}, I_{l+1}}\right\}_{l \in \mathbb{Z}}\right\|_{\ell^{2}} \\
& =\left\|\left\{2^{l+i} l^{k} \frac{1}{2^{l+1+i} \mathcal{A}^{-1}\left(\frac{2^{l+i+2}}{2|x|}\right)}\right\}_{l \in \mathbb{Z}}\right\|_{\ell^{2}} \\
& =\left\|\left\{\frac{l^{k}}{2 \mathcal{A}^{-1}\left(\frac{2^{l+i+1}}{|x|}\right)}\right\}_{l \in \mathbb{Z}}\right\|_{\ell^{2}} \\
& =\frac{1}{2}\left\|\left\{\frac{l^{k}}{\mathcal{A}^{-1}\left(\frac{2^{l+i+1}}{|x|}\right)}\right\}_{l \in \mathbb{Z}}\right\|_{\ell^{2}},
\end{aligned}
$$


which holds for all $|x|<2^{i}$. Then, taking supremum, we obtain

$$
\begin{aligned}
C_{\mathcal{A}} & \geq \sup _{2^{i-2}<|x|<2^{i-1}}\left\|\left\{\sum_{m=1}^{\infty} 2^{m} R m^{k}\left\|\left(K_{l}(\cdot-x)-K_{l}(\cdot)\right) \chi_{|y| \sim 2^{m} R}\right\|_{\mathcal{A}, B_{m+1}}\right\}_{l \in \mathbb{Z}}\right\|_{\ell^{2}} \\
& \geq \sup _{2^{i-2}<|x|<2^{i-1}} \frac{1}{2}\left\|\left\{\frac{l^{k}}{\mathcal{A}^{-1}\left(\frac{2^{l+i+1}}{|x|}\right)}\right\}_{l \in \mathbb{Z}}\right\|_{\ell^{2}} \geq \frac{1}{2}\left\|\left\{\frac{l^{k}}{\mathcal{A}^{-1}\left(2^{l} 8\right)}\right\}_{l \in \mathbb{Z}}\right\|_{\ell^{2}} .
\end{aligned}
$$

Hence,

$$
\left\|\left\{\frac{l^{k}}{\mathcal{A}^{-1}\left(2^{l} 8\right)}\right\}_{l \in \mathbb{Z}}\right\|_{\ell^{2}} \leq 2 C_{\mathcal{A}}<\infty .
$$

Proof of Corollary 4.5. Let $\mathcal{A}(t)=\exp \left(t^{1+k}\right)-1$. Using Proposition 4.4, it is enough to prove that for any $k \in \mathbb{N} \cup\{0\}$,

$$
\left\|\left\{\frac{l^{k}}{\mathcal{A}^{-1}\left(2^{l} 8\right)}\right\}_{l \in \mathbb{Z}}\right\|_{\ell^{2}}<\infty .
$$

As $\mathcal{A}(t)=\exp \left(t^{1+k}\right)-1$, we have that $\mathcal{A}^{-1}(t)=\log (t+1)^{k+1}$.

If $m=0, \mathcal{A}^{-1}\left(2^{m}\right)=\mathcal{A}^{-1}(1)=\log (1+1)^{k+1}=\log (2)^{k+1} \neq 0$, then $\frac{m^{k}}{\mathcal{A}^{-1}\left(2^{m} 8\right)}=0$.

Also, $\mathcal{A}^{-1}\left(2^{m} 8\right)=\log \left(2^{m} 8+1\right)^{k+1} \geq \log \left(2^{m} 8\right)^{k+1} \geq \log \left(2^{m}\right)^{k+1}$. Then, we get

$$
\begin{aligned}
\left\|\left\{\frac{m^{k}}{\mathcal{A}^{-1}\left(2^{m} 8\right)}\right\}_{m \in \mathbb{Z}}\right\|_{\ell^{2}}^{2} & =\sum_{m \in \mathbb{Z}}\left(\frac{m^{k}}{\mathcal{A}^{-1}\left(2^{m} 8\right)}\right)^{2}=\sum_{m \in \mathbb{Z} \backslash\{0\}}\left(\frac{m^{k}}{\mathcal{A}^{-1}\left(2^{m} 8\right)}\right)^{2} \\
\leq & \sum_{m \in \mathbb{Z} \backslash\{0\}}\left(\frac{m^{k}}{\log \left(2^{m}\right)^{k+1}}\right)^{2}=\sum_{m \in \mathbb{Z} \backslash\{0\}}\left(\frac{1}{\log (2)^{k+1}} \frac{m^{k}}{m^{k+1}}\right)^{2} \\
& =\frac{1}{\log (2)^{2(k+1)}} \sum_{m \in \mathbb{Z} \backslash\{0\}} \frac{1}{m^{2}}<\infty
\end{aligned}
$$

\section{Proofs of the MAIN RESUlts}

For the proof of the main results we need the following lemma.

Lemma 5.1. Let $k \in \mathbb{N} \cup\{0\}$. Let $\mathcal{A}, \mathcal{B}$ be Young functions such that $\overline{\mathcal{A}}^{-1}(t) \mathcal{B}^{-1}(t) \overline{\mathcal{C}}_{k}^{-1}(t) \leq t$, with $\overline{\mathcal{C}}_{k}(t)=\exp \left(t^{1 / k}\right)$ for $t \geq 1$. If $T$ is a vector-valued singular integral operator with kernel $K$ such that $K \in H_{\mathcal{B}, X}^{\dagger} \cap H_{\mathcal{A}, X, k}^{\dagger}$, then for any $b \in \mathrm{BMO}, 0<\delta<\varepsilon<1$ we have:

a) if $k=0, \mathcal{B}=\mathcal{A}$, then there exists $C>0$ such that

$$
M_{\delta}^{\sharp}\|T f\|_{\mathbf{x}}(x):=\left(M^{\sharp}\|T f\|_{\mathbf{x}}^{\delta}\right)^{\frac{1}{\delta}}(x) \leq C M_{\bar{A}} f(x),
$$

for all $x \in \mathbb{R}^{n}$.

b) If $k \in \mathbb{N}$, then there exists $C=C(\delta, \varepsilon)>0$ such that

$$
M_{\delta}^{\sharp}\left(\left\|T_{b}^{k} f\right\|_{X}\right)(x) \leq C \sum_{j=0}^{k-1}\|b\|_{\mathrm{BMO}}^{k-j} M_{\varepsilon}\left(T_{b}^{j} f\right)(x)+C\|b\|_{\mathrm{BMO}}^{k} M_{\overline{\mathcal{A}}} f(x),
$$

for all $x \in \mathbb{R}^{n}$. 
Proof. The argument is similar to the proof of Lemma 5.1 in [11; we only give the main changes. Let's consider part (b); the proof of part (a) is analogous, with $k=0$.

Let $K \in H_{\mathcal{B}, X}^{\dagger} \cap H_{A, X, k}^{\dagger}$ and $k \in \mathbb{N}$. Then for any $\lambda \in \mathbb{R}$, we can write

$$
T_{b}^{k} f(x)=T\left((\lambda-b)^{k} f\right)(x)+\sum_{m=0}^{k-1} C_{k, m}(b(x)-\lambda)^{k-m} T_{b}^{m} f(x)
$$

Let us fix $x \in \mathbb{R}^{n}$ and $B$ a ball such that $x \in B, \tilde{B}:=2 B$, and $c_{B}$ the center of the ball $B$. Let $f=f_{1}+f_{2}$, where $f_{1}:=f \chi_{\tilde{B}}$, and let $a:=\left\|T\left(b_{\tilde{B}}-b\right)^{k} f_{2}\left(c_{B}\right)\right\|_{X}$. Using 5.1 and taking $\lambda=b_{\tilde{B}}=\frac{1}{|\tilde{B}|} \int_{\tilde{B}} b$,

$$
\begin{gathered}
\left(\left.\frac{1}{|B|} \int_{B}\left|\left\|T_{b}^{k} f(y)\right\|_{X}^{\delta}-\right| a\right|^{\delta} \mid d y\right)^{1 / \delta} \leq\left(\frac{1}{|B|} \int_{B}\left\|T_{b}^{k} f(y)-T\left(b_{\tilde{B}}-b\right)^{k} f_{2}\left(c_{B}\right)\right\|_{X}^{\delta} d y\right)^{1 / \delta} \\
=\left(\frac{1}{|B|} \int_{B} \| \sum_{m=0}^{k-1} C_{k, m}\left(b(y)-b_{\tilde{B}}\right)^{k-m} T_{b}^{m} f(y)+T\left(\left(b_{\tilde{B}}-b\right)^{k} f\right)(y)\right. \\
\left.-T\left(\left(b_{\tilde{B}}-b\right)^{k} f_{2}\right)\left(c_{B}\right) \|_{X}^{\delta} d y\right)^{1 / \delta} \\
\leq C\left[\sum_{m=0}^{k-1} C_{k, m}\left(\frac{1}{|B|} \int_{B}\left\|\left(b(y)-b_{\tilde{B}}\right)^{k-m} T_{b}^{m} f(y)\right\|_{X}^{\delta} d y\right)^{1 / \delta}\right. \\
+\left(\frac{1}{|B|} \int_{B}\left\|T\left(\left(b_{\tilde{B}}-b\right)^{k} f_{1}\right)(y)\right\|_{X}^{\delta} d y\right)^{1 / \delta} \\
\left.+\left(\frac{1}{|B|} \int_{B}\left\|T\left(\left(b_{\tilde{B}}-b\right)^{k} f_{2}\right)(y)-T\left(\left(b_{\tilde{B}}-b\right)^{k} f_{2}\right)\left(c_{B}\right)\right\|_{X}^{\delta} d y\right)^{1 / \delta}\right] \\
=C[I+I I+I I I .
\end{gathered}
$$

The estimates of $I$ and $I I$ are analogous to the corresponding ones in [11, Lemma 5.1]. Then

$$
\begin{aligned}
& I \leq c \sum_{m=0}^{k-1} C_{k, m}\|b\|_{\mathrm{BMO}}^{k-m} M_{\varepsilon}\left(\left\|T_{b}^{k} f\right\|_{X}\right)(x), \\
& I I \leq C\|b\|_{\mathrm{BMO}}^{k} M_{\overline{\mathcal{A}}} f(x) .
\end{aligned}
$$


Now III. By Jensen's inequality and the property of the norm 2.1], we get

$$
\begin{aligned}
I I I & \leq \frac{1}{|B|} \int_{B}\left\|T\left(\left(b_{\tilde{B}}-b\right)^{k} f_{2}\right)(y)-T\left(\left(b_{\tilde{B}}-b\right)^{k} f_{2}\right)\left(c_{B}\right)\right\|_{X} d y \\
& =\frac{1}{|B|} \int_{B}\left\|\left\{\int_{\tilde{B}^{c}}\left(K_{l}(y-z)-K_{l}\left(c_{B}-z\right)\right)\left(b_{\tilde{B}}-b(z)\right)^{k} f(z) d z\right\}\right\|_{X} d y \\
& \leq \frac{1}{|B|} \int_{B}\left\|\left\{\left|\int_{\tilde{B}^{c}}\left(K_{l}(y-z)-K_{l}\left(c_{B}-z\right)\right)\left(b_{\tilde{B}}-b(z)\right)^{k} f(z) d z\right|\right\}\right\|_{X} d y \\
& \leq \frac{1}{|B|} \int_{B}\left\|\left\{\int_{\tilde{B}^{c}}\left|K_{l}(y-z)-K_{l}\left(c_{B}-z\right) \| b_{\tilde{B}}-b(z)\right|^{k}|f(z)| d z\right\}\right\| \|_{X} d y .
\end{aligned}
$$

For each coordinate $l \in \mathbb{Z}$, we proceed as in the proof of Lemma 5.1 in [11]. Let $B_{j}:=2^{j+1} B$, for $j \geq 1$, and we obtain

$$
\begin{aligned}
& \int_{\tilde{B}^{c}} \mid K_{l}(y-z)- K_{l}\left(c_{B}-z\right) \| b_{\tilde{B}}-\left.b(z)\right|^{k}|f(z)| d z \\
& \leq C\|b\|_{\mathrm{BMO}}^{k} M_{\overline{\mathcal{A}}} f(x)\left(\sum_{j=1}^{\infty}\left(2^{j} R\right)^{n}\left\|\left(K_{l}(y-\cdot)-K_{l}\left(c_{B}-\cdot\right)\right) \chi_{S_{j}}\right\|_{\mathcal{B}, B_{j}}\right. \\
&\left.\quad+\sum_{j=1}^{\infty}\left(2^{j} R\right)^{n} j^{k}\left\|\left(K_{l}(y-\cdot)-K_{l}\left(c_{B}-\cdot\right)\right) \chi_{S_{j}}\right\|_{\mathcal{A}, B_{j}}\right) .
\end{aligned}
$$

Hence,

$$
\begin{aligned}
& I I I \leq \frac{1}{|B|} \int_{B} \|\left\{C \| b \| _ { \mathrm { BMO } } ^ { k } M _ { \overline { \mathcal { A } } } f ( x ) \left(\sum_{j=1}^{\infty}\left(2^{j} R\right)^{n}\left\|\left(K_{l}(y-\cdot)-K_{l}\left(c_{B}-\cdot\right)\right) \chi_{S_{j}}\right\|_{\mathcal{B}, B_{j}}\right.\right. \\
&\left.\left.+\sum_{j=1}^{\infty}\left(2^{j} R\right)^{n} j^{k}\left\|\left(K_{l}(y-\cdot)-K_{l}\left(c_{B}-\cdot\right)\right) \chi_{S_{j}}\right\|_{\mathcal{A}, B_{j}}\right)\right\} \|_{X} d y \\
& \leq C\|b\|_{\mathrm{BMO}}^{k} M_{\overline{\mathcal{A}}} f(x) \frac{1}{|B|} \int_{B}\left[\left\|\sum_{j=1}^{\infty}\left(2^{j} R\right)^{n}\right\|\left(K_{l}(y-\cdot)-K_{l}\left(c_{B}-\cdot\right)\right) \chi_{S_{j}}\left\|_{\mathcal{B}, B_{j}}\right\|_{X}\right. \\
&\left.\quad+\left\|\sum_{j=1}^{\infty}\left(2^{j} R\right)^{n} j^{k}\right\|\left(K_{l}(y-\cdot)-K_{l}\left(c_{B}-\cdot\right)\right) \chi_{S_{j}}\left\|_{\mathcal{A}, B_{j}}\right\|_{X}\right] d y \\
& \leq C\|b\|_{\mathrm{BMO}}^{k} M_{\overline{\mathcal{A}}} f(x) \frac{1}{|B|} \int_{B} d y=C\|b\|_{\mathrm{BMO}}^{k} M_{\overline{\mathcal{A}}} f(x),
\end{aligned}
$$

where the last inequality holds since $K \in H_{\mathcal{B}, X}^{\dagger} \cap H_{\mathcal{A}, X, k}^{\dagger}$ and we have used that $x \in B \subset B_{j}$ and that $\left|x_{B}-y\right|<R$ since $y \in B$.

Thus,

$$
\begin{array}{r}
\left(\left.\frac{1}{|B|} \int_{B}\left|\left\|T_{b}^{k}(y)\right\|_{X}^{\delta}-\right| a\right|^{\delta} \mid d y\right)^{1 / \delta} \leq C \sum_{m=0}^{k-1} C_{k, m}\|b\|_{\mathrm{BMO}}^{k-m} M_{\varepsilon}\left(\left\|T_{b}^{k} f\right\|_{X}\right)(x) \\
+C\|b\|_{\mathrm{BMO}}^{k} M_{\overline{\mathcal{A}}} f(x)
\end{array}
$$


Now we proceed to prove the main theorems.

Proof of Theorem 3.3. By the extrapolation result Theorem 1.1 in [5], estimate (3.1) holds for all $0<p<\infty$ and all $w \in A_{\infty}$ if, and only if, it holds for some $0<p_{0}<\infty$ and all $w \in A_{\infty}$. Therefore, we will show that (3.1) is true for $p_{0}$, which is taken such that $T$ is of strong type $\left(p_{0}, p_{0}\right)$.

First we consider $w \in L^{\infty} \cap A_{\infty}$. Since $w \in A_{\infty}$, there exists $r>1$ such that $w \in A_{r}$. Observe that for $0<\delta=p_{0} / r<1, w \in A_{p_{0} / \delta}$. Since $f \in L_{c}^{\infty}$, we have

$$
\left\|M_{\delta}(T f)\right\|_{L^{p_{0}(w)}}=\left\|M\left(|T f|^{\delta}\right)\right\|_{L^{\frac{p_{0}}{\delta}(w)}}^{\frac{1}{\delta}} \leq C\|T f\|_{L^{p_{0}(w)}} \leq\|w\|_{L^{\infty}}\|T f\|_{L^{p_{0}}}<\infty .
$$

Then using part (a) of Lemma 5.1 we obtain

$$
\begin{aligned}
\int_{\mathbb{R}^{n}}\|T f\|_{\mathbf{x}}^{p_{0}} w & \leq \int_{\mathbb{R}^{n}} M_{\frac{1}{r}}\left(\|T f\|_{\mathbf{x}}^{p_{0}}\right) w=\int_{\mathbb{R}^{n}}\left(M\left(\|T f\|_{\mathbf{x}}^{p_{0} / r}\right)\right)^{r} w \\
& \leq c \int_{\mathbb{R}^{n}}\left(M^{\sharp}\left(\|T f\|_{\mathbf{x}}^{\delta}\right)\right)^{p_{0} / \delta} w=c \int_{\mathbb{R}^{n}}\left(M_{\delta}^{\sharp}\left(\|T f\|_{\mathbf{x}}\right)^{p_{0}} w\right. \\
& \leq c \int_{\mathbb{R}^{n}}\left(M_{\bar{A}} f\right)^{p_{0}} w .
\end{aligned}
$$

Thus, for all $w \in L^{\infty} \cap A_{\infty}$ we have 3.1 for $p_{0}$, that is

$$
\int_{\mathbb{R}^{n}}\|T f\|_{\mathbf{x}}^{p_{0}} w \leq c \int_{\mathbb{R}^{n}}\left(M_{\bar{A}} f\right)^{p_{0}} w
$$

If we consider $w \in A_{\infty}$, for any $N>0$ we define $w_{N}=\min \{w, N\}$. Then $w_{N} \in A_{\infty}$ and also $\left[w_{N}\right]_{A_{\infty}} \leq C[w]_{A_{\infty}}$ with $C$ independent of $N$. Since $w_{N} \in L^{\infty}$, (3.1) holds for $w_{N}$ and $C$ does not depend on $N$. Letting $N \rightarrow \infty$ and using the monotone convergence theorem we conclude (3.1) for $p_{0}$ and any $w \in A_{\infty}$.

Thus, as mentioned, using the extrapolation results obtained in [5], 3.1) holds for all $0<p<\infty$ and $w \in A_{\infty}$.

Proof of Theorem 3.4. The proof is analogous to the proof of Theorem 3.3, part (a), in [11], using in this case Lemma 5.1.

\section{Fractional integrals}

For fractional integral operators there exist $L^{\mathcal{A}, \alpha}$-Hörmander conditions defined in [2]. The authors obtained the inequality 1.1 with $M_{\overline{\mathcal{A}}, \alpha}$, the fractional maximal operator associated to $\overline{\mathcal{A}}$. In this section, we present a weaker condition for fractional vector-valued kernels and obtain similar results and applications.

Recall the notation: $|x| \sim s$ means $s<|x| \leq 2 s$ and given a Young function $\mathcal{A}$ we write $\|f\|_{\mathcal{A},|x| \sim s}=\left\|f \chi_{|x| \sim s}\right\|_{\mathcal{A}, B(0,2 s)}$.

The new condition is the following.

Definition 6.1. Let $K_{\alpha}=\left\{K_{\alpha, l}\right\}_{l \in \mathbb{Z}}$ be a vector-valued function, $\mathcal{A}$ be a Young function, $0<\alpha<n$, and $k \in \mathbb{N} \cup\{0\}$. The function $K_{\alpha}$ satisfies the $L_{\dagger}^{\alpha, \mathcal{A}, X, k}$ Hörmander condition $\left(K \in H_{\alpha, \mathcal{A}, X, k}^{\dagger}\right)$, if there exist $c_{\mathcal{A}}>1$ and $C_{\mathcal{A}}>0$ such that 
for all $x$ and $R>c_{\mathcal{A}}|x|$,

$$
\left\|\left\{\sum_{m=1}^{\infty}\left(2^{m} R\right)^{n-\alpha} m^{k}\left\|K_{\alpha, l}(\cdot-x)-K_{\alpha, l}(\cdot)\right\|_{\mathcal{A},|y| \sim 2^{m} R}\right\}_{l \in \mathbb{Z}}\right\|_{X} \leq C_{\mathcal{A}} .
$$

We say that $K_{\alpha} \in H_{\alpha, \infty, k}^{\dagger}$ if $K_{\alpha}$ satisfies the previous condition with $\|\cdot\|_{L^{\infty},|x| \sim 2^{m} R}$ in place of $\|\cdot\|_{\mathcal{A},|x| \sim 2^{m} R}$.

If $k=0$, we denote $H_{\alpha, \mathcal{A}, X}^{\dagger}=H_{\alpha, \mathcal{A}, X, 0}^{\dagger}$ and $H_{\alpha, \infty, X}^{\dagger}=H_{\alpha, \infty, X, 0}^{\dagger}$.

We also need an extra condition that ensures a certain control of the size; in this case it is:

Definition 6.2. Let $\mathcal{A}$ be a Young function and let $0<\alpha<n$. The function $K_{\alpha}=\left\{K_{\alpha, l}\right\}_{l \in \mathbb{Z}}$ is said to satisfy the $\mathscr{S}_{\alpha, \mathcal{A}, X}^{\dagger}$ condition, denoted by $K_{\alpha} \in \mathscr{S}_{\alpha, \mathcal{A}, X}^{\dagger}$, if there exists a constant $C>0$ such that

$$
\left\|\left\{\left\|K_{\alpha, l}\right\|_{\mathcal{A},|x| \sim s}\right\}_{l \in \mathbb{Z}}\right\|_{X} \leq C s^{\alpha-n} .
$$

Remark 6.3. If $\mathcal{A}(t) \leq c \mathcal{B}(t)$ for $t>t_{0}$, some $t_{0}>0$, then

$$
H_{\alpha, \mathcal{B}, X, k}^{\dagger} \subset H_{\alpha, \mathcal{A}, X, k}^{\dagger} \quad \text { and } \quad \mathscr{S}_{\alpha, \mathcal{B}, X}^{\dagger} \subset \mathscr{S}_{\alpha, \mathcal{A}, X}^{\dagger} \cdot
$$

Remark 6.4. Observe that the $M_{\alpha, \overline{\mathcal{A}}}$ is the fractional maximal operator associated to the Young function $\overline{\mathcal{A}}$, that is

$$
M_{\alpha, \overline{\mathcal{A}}} f(x):=\sup _{B \ni x}|B|^{\alpha / n}\|f\|_{\overline{\mathcal{A}}, B} .
$$

The results in this case are

Theorem 6.5. Let $\mathcal{A}$ be a Young function and $0<\alpha<n$. Let $T_{\alpha} f=\left\{K_{\alpha, l} * f\right\}_{l \in \mathbb{Z}}$ with kernel $K_{\alpha}=\left\{K_{\alpha, l}\right\}_{l \in \mathbb{Z}} \in \mathscr{S}_{\alpha, \mathcal{A}, X}^{\dagger} \cap H_{\alpha, \mathcal{A}, X}^{\dagger}$. Let $0<p<\infty$ and $w \in A_{\infty}$. Then there exists $c>0$ such that

$$
\int_{\mathbb{R}^{n}}\left\|T_{\alpha} f\right\|_{X}^{p} w \leq C \int_{\mathbb{R}^{n}}\left(M_{\alpha, \overline{\mathcal{A}}} f\right)^{p} w, \quad f \in L_{c}^{\infty}\left(\mathbb{R}^{n}\right),
$$

whenever the left-hand side is finite.

Theorem 6.6. Let $0<\alpha<n, b \in \mathrm{BMO}$ and $k \in \mathbb{N}$. Let $T_{\alpha}$ be a convolution operator with kernel $K_{\alpha}=\left\{K_{\alpha, l}\right\}_{l \in \mathbb{Z}}$ such that $T_{\alpha}$ is bounded from $L_{X}^{p_{0}}(d x)$ to $L_{X}^{q_{0}}(d x)$, for some $1<p_{0}, q_{0}<\infty$. Let $\mathcal{A}, \mathcal{B}$ be Young functions such that $\overline{\mathcal{A}}^{-1}(t) \mathcal{B}^{-1}(t) \overline{\mathcal{C}}_{k}^{-1}(t) \leq t$, with $\overline{\mathcal{C}}_{k}(t)=\exp \left(t^{1 / k}\right)$ for $t \geq 1$. If $K_{\alpha} \in \mathscr{S}_{\alpha, \mathcal{A}, X}^{\dagger} \cap$ $H_{\alpha, \mathcal{A}, X}^{\dagger} \cap H_{\alpha, \mathcal{B}, X, k}^{\dagger}$, then for any $0<p<\infty$ and any $w \in A_{\infty}$, there exists $c>0$ such that

$$
\int_{\mathbb{R}^{n}}\left\|T_{\alpha, b}^{k} f\right\|_{X}^{p} w \leq C\|b\|_{\mathrm{BMO}}^{p k} \int_{\mathbb{R}^{n}}\left(M_{\alpha, \overline{\mathcal{A}}} f\right)^{p} w, \quad f \in L_{c}^{\infty}\left(\mathbb{R}^{n}\right),
$$

whenever the left-hand side is finite. 
Remark 6.7. The proofs of these results are analogous to the ones in [2] with the same changes of the results for the vector-valued singular integral operators above. Also for the proofs of these results we need the following lemma, whose proof is analogous to that of Lemma 5.1] and that of Theorem 3.6 in [2].

Lemma 6.8. Let $\mathcal{A}$ be a Young function and $0<\alpha<n$. Let $T_{\alpha} f=K_{\alpha} * f$ with kernel $K_{\alpha} \in \mathscr{S}_{\alpha, \mathcal{A}, X}^{\dagger} \cap H_{\alpha, \mathcal{A}, X}^{\dagger}$. Then for all $0<\delta<\varepsilon<1$ there exists c $>0$ such that

$$
M_{\delta}^{\sharp}\left\|T_{\alpha} f\right\|_{\mathbf{x}}(x)=\left(M^{\sharp}\left\|T_{\alpha} f\right\|_{X}^{\delta}\right)^{\frac{1}{\delta}}(x) \leq c M_{\alpha, \overline{\mathcal{A}}} f(x),
$$

for all $x \in \mathbb{R}^{n}$ and $f \in L_{c}^{\infty}$.

There exist relations between the kernels which satisfy the fractional conditions $\mathscr{S}_{\alpha, \mathcal{A}, X}^{\dagger}$ and $H_{\alpha, \mathcal{A}, X, k}^{\dagger}$ and the kernels which satisfy the conditions $\mathscr{S}_{\mathcal{A}, X}^{\dagger}$ and $H_{\mathcal{A}, X, k}^{\dagger}$. The next proposition shows this relation and also a form to define kernels such that they satisfy the fractional condition. The proof is analogous to that of Proposition 4.1 in [2].

Proposition 6.9. Let $K=\left\{K_{l}\right\}_{l \in \mathbb{Z}}$ and $K_{\alpha}=\left\{K_{\alpha, l}\right\}_{l \in \mathbb{Z}}$ defined by $K_{\alpha}(x)=$ $|x|^{\alpha} K(x)$. If $K \in \mathscr{S}_{\mathcal{A}, X}^{\dagger} \cap H_{\mathcal{A}, X, k}^{\dagger}$ then $K_{\alpha} \in \mathscr{S}_{\alpha, \mathcal{A}, X}^{\dagger} \cap H_{\alpha, \mathcal{A}, X, k}^{\dagger}$.

We know that, for certain Banach spaces $X$, the kernel of the square operator satisfies the conditions $\mathscr{S}_{\mathcal{A}, X}^{\dagger}$ and $H_{\mathcal{A}, X, k}^{\dagger}$, for example $X=\ell^{p}$ and $\mathcal{A}(t)=\exp ^{\frac{1}{1+k}}-1$; for more examples see Section 4 . Now we can define the fractional square operator,

$$
S_{\alpha, X} f(x):=\left\|\tilde{T}_{\alpha} f(x)\right\|_{X}=\left\|\left\{\int_{\mathbb{R}}|x-y|^{\alpha} K_{l}(x-y) f(y) d y\right\}\right\|_{X},
$$

where $K=\left\{K_{l}\right\}_{l \in \mathbb{Z}}$ is the kernel defined in Section 4. Let $b \in \mathrm{BMO}$ and $k \in \mathbb{N}$; the commutator is defined by

$S_{\alpha, X, b}^{k} f(x):=\left\|\tilde{T}_{\alpha, b}^{k} f(x)\right\|_{X}=\left\|\left\{\int_{R}(b(x)-b(y))^{k}|x-y|^{\alpha} K_{l}(x-y) f(y) d y\right\}_{l \in \mathbb{Z}}\right\|_{X}$.

By Proposition 6.9, we have that $S_{\alpha, X} f(x)$ satisfies the hypothesis of Theorem 6.6 Then, Theorem 3.6 for the fractional square operator is

Theorem 6.10. Let $b \in \mathrm{BMO}, k \in \mathbb{N} \cup\{0\}$, and $0<\alpha<n$. Let $\mathcal{A}(t)=$ $\exp \left(t^{\frac{1}{1+k}}\right)-1$. If $K \in \mathscr{S}_{\mathcal{A}, X}^{\dagger} \cap H_{\mathcal{A}, X, k}^{\dagger}$, i.e.

$$
\left\|\left\{\frac{1}{m}\right\}_{m \in(\mathbb{Z}-\{0\})}\right\|_{X}=C_{\mathcal{A}, X}<\infty
$$

then, for any $0<p<\infty$ and $w \in A_{\infty}$, there exists $C$ such that

$$
\int_{\mathbb{R}^{n}}\left|S_{\alpha, X, b}^{k} f(x)\right|^{p} w(x) d x \leqslant C \int_{\mathbb{R}^{n}}\left(M_{\alpha, L \log L^{k+1}} f(x)\right)^{p} w(x) d x .
$$

In [1], the authors studied the weights for the fractional maximal operator related to a Young function in the context of variable Lebesgue spaces. They characterized the weights for the boundedness of $M_{\alpha, \mathcal{A}}$ with $\mathcal{A}(t)=t^{r}(1+\log (t))^{\beta}, r \geq 1$ and $\beta \geq 0$. 
For any $1 \leq p, q<\infty$, we define the $A_{p, q}$ weight class this way: $w \in A_{p, q}$ if and only if $w^{q} \in A_{1+\frac{q}{p^{\prime}}}$.

The result in the classical Lebesgue spaces, that is, the variable Lebesgue spaces with constant exponent, is the following.

Theorem I ([1]). Let $w$ be a weight, $0<\alpha<n, 1<p<n / \alpha$, and $1 / q=1 / p-\alpha / n$. Let $\mathcal{A}(t)=t^{r}(1+\log (t))^{\beta}$, with $1 \leq r<p$ and $\beta \geq 0$. Then $M_{\alpha, \mathcal{A}}$ is bounded from $L^{p}\left(w^{p}\right)$ into $L^{q}\left(w^{q}\right)$ if and only if $w^{r} \in A_{p / r, q / r}$.

Applying this result to Theorem 6.10 we obtain that if $w \in A_{p, q}$ then for all $1<p<n / \alpha$ and $1 / q=1 / p-\alpha / n$,

$$
\begin{aligned}
\int_{\mathbb{R}^{n}}\left|S_{\alpha, X, b}^{k} f(x)\right|^{q} w^{q}(x) d x & \leq c \int_{\mathbb{R}^{n}}\left(M_{\alpha, L \log L^{k+1}} f(x)\right)^{q} w^{q}(x) d x \\
& \leq c \int_{\mathbb{R}^{n}}|f(x)|^{p} w^{p}(x) d x .
\end{aligned}
$$

So we have the following result.

Corollary 6.11. Let $0<\alpha<1,1<p<1 / \alpha$, and $1 / q=1 / p-\alpha$. If $w \in A_{p, q}$ then $S_{\alpha, X, b}^{k}$ is bounded from $L^{p}\left(w^{p}\right)$ into $L^{q}\left(w^{q}\right)$.

\section{REFERENCES}

[1] A. Bernardis, E. Dalmasso, G. Pradolini, Generalized maximal functions and related operators on weighted Musielak-Orlicz spaces, Ann. Acad. Sci. Fenn. Math. 39 (2014), no. 1, 23-50. MR 3186804.

[2] A. L. Bernardis, M. Lorente, M. S. Riveros Weighted inequalities for fractional integral operators with kernel satisfying Hörmander type conditions, Math. Inequal. Appl. 14 (2011), no. 4, 881-895. MR 2884902.

[3] R. R. Coifman, Distribution function inequalities for singular integrals, Proc. Nat. Acad. Sci. U.S.A. 69 (1972), 2838-2839. MR 0303226

[4] R. R. Coifman, R. Rochberg, G. Weiss Factorization theorems for Hardy spaces in several variables, Ann. of Math. (2) 103 (1976), no. 3, 611-635. MR 0412721

[5] D. Cruz-Uribe, J. M. Martell, C. Pérez, Extrapolation from $A_{\infty}$ weights and applications, J. Funct. Anal. 213 (2004), no. 2, 412-439. MR 2078632

[6] J. Duoandikoetxea, Fourier Analysis. Graduate Texts in Mathematics, 29, American Mathematical Society, 2001. MR 1800316

[7] N. Fava, F. Zó, Medida e integral de Lebesgue, Red Olímpica, Buenos Aires, 1996.

[8] C. Fefferman, E. M. Stein, $H^{p}$ spaces of several variables, Acta Math. 129 (1972), no. 3-4, 137-193. MR 0447953

[9] L. Grafakos, Modern Fourier Analysis, 2nd edition, Graduate Texts in Mathematics, 250, Springer, 2009. MR 2463316.

[10] D. S. Kurtz, R. L. Wheeden, Results on weighted norm inequalities for multipliers, Trans. Amer. Math. Soc. 255 (1979), 343-362. MR 0542885

[11] M. Lorente, J. M. Martell, M. S. Riveros, A. de la Torre, Generalized Hörmander's conditions, commutators and weights, J. Mathe. Anal. Appl. 342 (2008), no. 2, 1399-1425. MR 2445285

[12] M. Lorente, M. S. Riveros, A. de la Torre, Weighted estimates for singular integral operators satisfying Hörmander's conditions of Young type, J. Fourier Anal. Appl. 11 (2005), no. 5, 497-509. MR 2182632

[13] M. Lorente, M. S. Riveros, A. de la Torre, On the Coifman type inequality for the oscillation of the one-sided averages, J. Math. Anal. Appl. 336 (2007), no. 1, 577-592. MR 2348527 
[14] J. M. Martell, C. Pérez, R. Trujillo-González, Lack of natural weighted estimates for some singular integral operators, Trans. Amer. Math. Soc. 357 (2005), no. 1, 385-396. MR 2098100.

[15] R. O'Neil, Fractional integration in Orlicz spaces. I, Trans. Amer. Math. Soc. 115 (1965), 300-328. MR 0194881.

[16] J. L. Rubio de Francia, F. J. Ruiz, J. L. Torrea, Calderón-Zygmund theory for operatorvalued kernels, Adv. in Math. 62 (1986), no. 1, 7-48. MR 0859252

[17] A. de la Torre, J. L. Torrea, One-sided discrete square function, Studia Math. 156 (2003), 243-260. MR 1978442

[18] F. C. Claassens, Combination of Orlicz norms, Bachelorscriptie, 17 Januari 2013, Mathematisch Instituut, Universiteit Leiden.https://www.math.leidenuniv.nl/scripties/ BachClaassens.pdf

\author{
A. L. Gallo \\ FaMAF, Universidad Nacional de Córdoba \\ CIEM (CONICET) \\ 5000 Córdoba, Argentina \\ andregallo88@gmail.com \\ G. H. Ibañez Firnkorn ${ }^{凶}$ \\ FaMAF, Universidad Nacional de Córdoba \\ CIEM (CONICET) \\ 5000 Córdoba, Argentina \\ gibanez@famaf .unc.edu.ar \\ M. S. Riveros \\ FaMAF, Universidad Nacional de Córdoba \\ CIEM (CONICET) \\ 5000 Córdoba, Argentina \\ sriveros@famaf.unc.edu.ar
}

Received: May 15, 2018

Accepted: November 10, 2018 